ISSN electrónico: 2445-1355

DOI: http://dx.doi.org/10.14201/fj2019421929

\title{
PERFIL DEL PACIENTE AMBULATORIO EN TRATAMIENTO CON ANSIOLÍTICOS Y/O ANTIDEPRESIVOS
}

\section{Outpatient Profile in Anxiolytic and/or Antidepressant Treatment}

\section{Laína OYAGUE LÓPEZ; Ramona MATEOS CAMPOS}

Departamento de Ciencias Biomédicas y del Diagnóstico. Área de Medicina Preventiva y Salud Pública. Facultad de Medicina. Universidad de Salamanca

Campus Miguel de Unamuno C/ Alfonso X el Sabio, s/n, 37007 Salamanca

Correo-e: mateos@usal.es

RESUMEN: Tanto los fármacos ansiolíticos, benzodiacepinas hipnóticas como antidepresivos han experimentado un notable aumento en su consumo durante los últimos años. Debido a su actividad a nivel central son ampliamente utilizados en el tratamiento de patologías como insomnio, ansiedad o depresión. El objetivo de este trabajo es conocer el perfil del paciente ambulatorio en tratamiento con alguno de estos principios activos. Para ello se ha llevado a cabo un estudio epidemiológico descriptivo transversal, para el que fue necesaria la resolución de un cuestionario por parte de aquellos pacientes que cumplieran los criterios de inclusión del estudio. Para su elaboración se consultaron las fichas técnicas de todos los principios activos comercializados en España pertenecientes al grupo de ansiolíticos, benzodiacepinas hipnóticas o antidepresivos. Se recogieron un total de 104 cuestionarios cuyos datos fueron almacenados y analizados en una base de datos. Se observó como el consumo de ansiolíticos y antidepresivos es más frecuente en mujeres y aumenta con la edad, siendo mayor el consumo de ansiolíticos en personas jubiladas y el de antidepresivos en trabajadoras en activo, y, en general, los tratamientos son de larga duración. Lorazepam es el fármaco ansiolítico más prescrito, mientras que Escitalopram lo es para el grupo de antidepresivos. 
Palabras clave: Ansiolítico; Antidepresivo; Perfil del Paciente Ambulatorio; Lorazepam; Escitalopram.

\begin{abstract}
Prescription of anxiolytic, hypnotic benzodiazepines and antidepressant drugs has experienced a remarkable increase during recent years. Due to their central effects, these drugs are currently being widley used in the treatment of pathologies such as insomnia, anxiety or depression. The object of this study is to draw the profile of outpatients undergoing treatment with any of the aforementioned active principles. Thus, a cross-sectional descriptive epidemiological study was carried out. In this context, patients meeting the study's inclusión criteria were invited to fill in a questionnaire. During the questionnaire design phase, all technical specifications of the active principles being commercialised in Spain wich fall within the anxiolytic, hypnotic benzodizepines or antidepressant groups were consulted. A total of 104 questionnaires were collected and data compiled and analyzed using a database system. It was noted that the consumption of anxiolytic and antidepressant drugs is more frequent among female patients and that it increases with patients age. Retired people are the greatest consumers of anxiolytic drugs and so are active workers for the antidepressant group. In general, they are long-term treatments. Lorazepam is the anxiolytic drug most frequently prescribed, while Escitalopram is the top one within the antidepressant group.
\end{abstract}

Key words: Anxiolytic; Antidepressant; Outpatient Profile; Lorazepam; Escitalopram.

\title{
1. INTRODUCCIÓN
}

Los fármacos ansiolíticos (N05B) (WHO, 2009) engloban una amplia oferta de principios activos, siendo las benzodiacepinas el subgrupo mayoritario, que facilitan la apertura del canal del GABA (Lorenzo et al., 2009) y también forman parte del grupo de fármacos hipnótico-sedantes (N05CD, N05CF).

Los medicamentos antidepresivos (N06A), dentro de los cuales también existe una gran oferta, están encaminados a la elevación de los niveles centrales de monoaminas.

El consumo de fármacos ansiolíticos y benzodiacepinas hipnóticas, y antidepresivos ha aumentado en España durante los últimos años. El consumo de los primeros se incrementó un 57,4 \% durante el periodo 2000-2012 (AEMPS, 2014), mientras que el de los antidepresivos fue del $200 \%$ durante la década 2003-2013

Ediciones Universidad de Salamanca / @@ఠ $\quad$ FarmaJournal, vol. 4, núm. 2 (2019), pp. 19-29 
(AEMPS, 2015). Ambos grupos farmacológicos comprenden una gran variedad de principios activos, siendo los más consumidos Lorazepam y Zolpidem, como ansiolíticos e hipnóticos, y Escitalopram y Venlafaxina como antidepresivos.

\section{Oвjetivos}

\subsection{Objetivo General}

Conocer el perfil del paciente en tratamiento con ansiolíticos y/o antidepresivos.

\subsection{Objetivos Especificos}

1. Analizar el perfil del paciente teniendo en cuenta variables epidemiológicas de persona: edad, sexo, nivel de estudios, profesión o situación laboral.

2. Valorar cuáles son los ansiolíticos y antidepresivos habitualmente prescritos.

3. Estudiar la frecuencia de aparición de efectos adversos.

4. Identificar los pacientes de nuevo diagnóstico.

5. Analizar las patologías concomitantes y tratamientos simultáneos.

\section{MATERIAles y MÉTOdos}

1. Tipo de estudio: Estudio epidemiológico descriptivo transversal (Piédrola Gil, 2015).

2. Población: Como criterio de inclusión se seleccionaron pacientes en tratamiento con al menos un ansiolítico, benzodiacepina hipnótica o antidepresivo, siendo un total de 104 participantes. Se seleccionaron en la oficina de farmacia, en el momento de la dispensación de alguno de estos fármacos, donde decidieron libremente responder al cuestionario.

3. Recogida de Datos. Se realizó a través de la aplicación de un cuestionario. Para elaborar el cuestionario se realizó una búsqueda de todos los principios activos pertenecientes a cada grupo de estudio, así como de las especialidades comercializadas. Las fichas técnicas (AEMPS, 2018) de cada una de ellas fueron consultadas para poder elaborar las opciones de respuesta del cuestionario.

4. Variables de estudio:

- Variables Epidemiológicas de Persona:

- Sexo.

- Edad.

- Nivel de Estudios. 
LAIINA OYAGUE LÓPEZ Y RAMONA MATEOS CAMPOS

PERFIL DEL PACIENTE AMBULATORIO EN TRATAMIENTO CON ANSIOLÍTICOS Y/O ANTIDEPRESIVOS

- Profesión.

- Situación Laboral.

- Variables Dependientes:

- Tipo de Medicamento.

- Dosis.

- Condición de uso: crónico o agudo.

- Indicación.

- Duración del Tratamiento.

- Efectos Adversos.

- Adherencia.

- Patologías y Medicación Adicionales.

- Pacientes de Nuevo Diagnóstico: un mes o menos de tratamiento

5. Se realizó un estudio piloto para elaborar el cuestionario definitivo.

6. Aplicación del Cuestionario mediante entrevista, siempre realizada por la misma persona, durante marzo de 2018.

Análisis de los resultados. Se creó una base de datos en Microsoft Excel, a partir de la cual se calcularon las frecuencias y porcentajes para elaborar las correspondientes tablas y figuras. Las diferencias entre variables se estudiaron mediante el estadístico «chi cuadrado».

\section{Resultados}

\subsection{Características de los pacientes}

El 85,6 \% seguía un tratamiento con ansiolíticos o benzodiacepinas hipnóticas, de los cuales el 89,9\% estaba tratado con, al menos, un ansiolítico, mientras que el 19,1 \% lo hacía con, al menos, una benzodiacepina hipnótica. A su vez, el 50,0 \% de los participantes seguía un tratamiento con antidepresivos y el 35,6 \% tomaba fármacos pertenecientes a ambos grupos terapéuticos (Figura 1).

Según el sexo, el 70,2 \% de los pacientes son mujeres, siendo mayoría en cualquier rango de edad. La prescripción de ansiolíticos y benzodiacepinas hipnóticas también aumenta con los años, siendo la proporción mayor, personas con 70 o más $(33,7 \%)$. En cambio, los antidepresivos son más utilizados entre los 50-59 años $(30,8 \%)$.

Los estudios secundarios son los más comunes para el grupo de ansiolíticos y benzodiacepinas hipnóticas (34,8\%), mientras que para pacientes en tratamiento con combinación $(37,8 \%)$ su frecuencia es idéntica a la de estudios primarios, siendo estos últimos los que predominan en pacientes tratados con antidepresivos $(38,5 \%)$.

Ediciones Universidad de Salamanca / @@ఠ $\quad$ FarmaJournal, vol. 4, núm. 2 (2019), pp. 19-29 
Figura 1. Distribución de Pacientes en función del Medicamento utilizado y según el Sexo (\%).

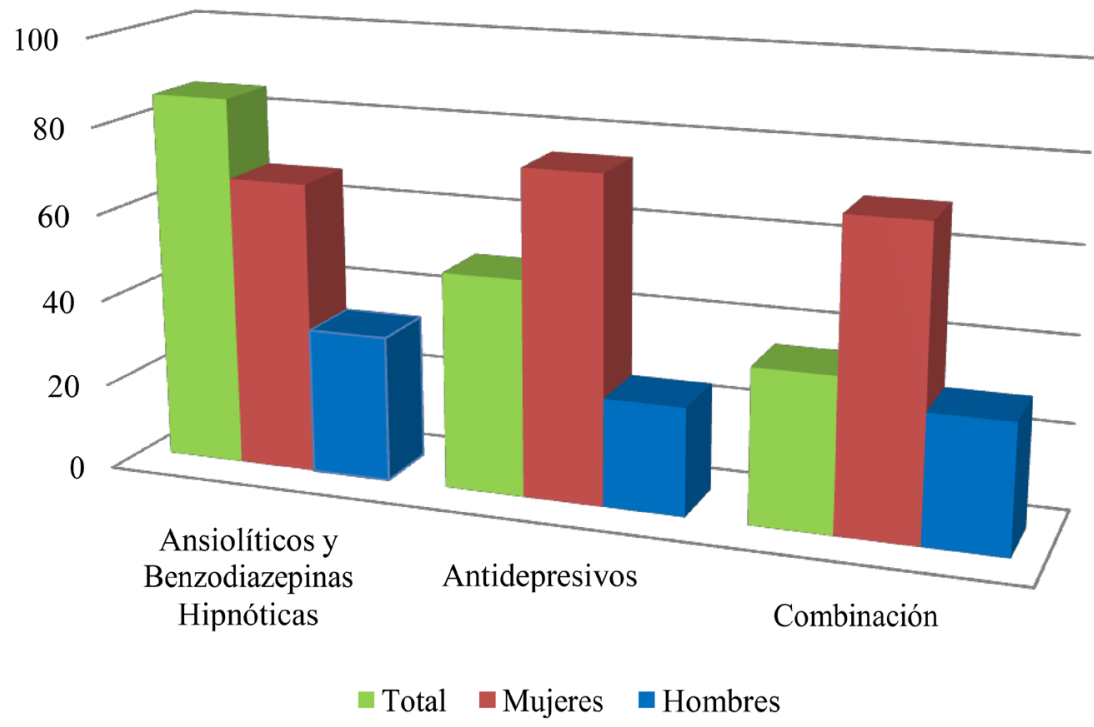

El grupo profesional más común son los asalariados (42,3\%) seguidos de aquellas personas que desarrollan otras actividades no remuneradas (40,8 \%).

La actividad laboral predominante es la jubilación (Ansiolíticos 47,2 \%; Combinación 35,1\%), seguido de los trabajadores en activo, siendo estos mayoritarios en pacientes tratados con antidepresivos (32,7 \%).

\subsection{Tipo de medicamento utilizado}

Las benzodiacepinas (Bzd) suponen 99,0 \% de fármacos ansiolíticos e hipnóticos prescritos, siendo las de acción ansiolítica las predominantes (83,6\%). Lorazepam (40,4 \%) es el más prescrito, seguido de Alprazolam (16,3\%) y Bromazepam (14,4\%). Las benzodiacepinas hipnóticas representaron el 16,4 $\%$, siendo Lormetazepam el más empleado $(9,6 \%)$. En combinación con antidepresivos Lorazepam $(54,1 \%)$ y Alprazolam $(27,0 \%)$ son los más utilizados. En las combinaciones entre ansiolíticos o benzodiacepinas hipnóticas, el más prescrito es Lorazepam (69,2 \%), seguido de Lormetazepam (46,2 \%) (Figura 2). 
Figura 2. Consumo de Principios Activos Ansiolíticos

y Benzodiazepinas Hipnóticas (\%).

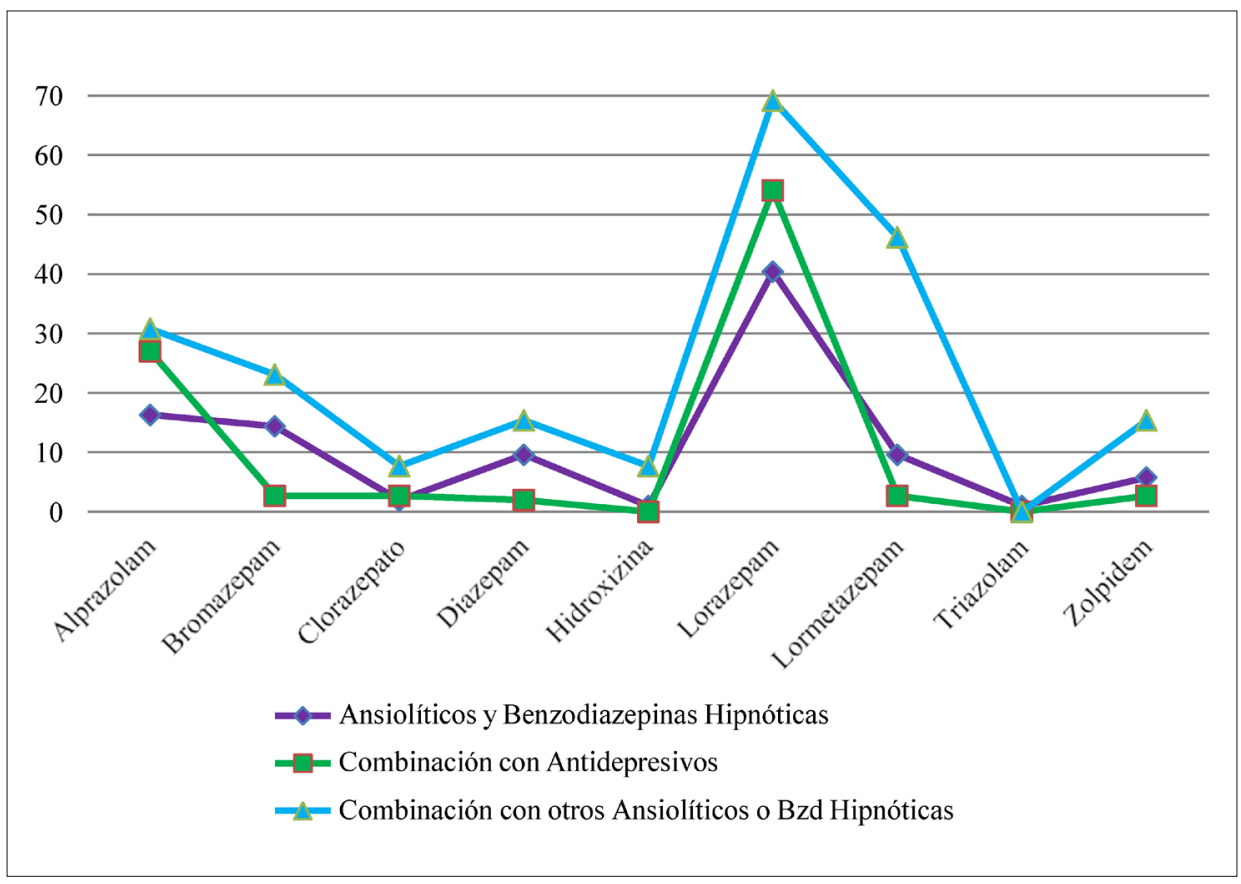

El consumo de Lorazepam es mayoritario en mujeres (47,9\%), mientras que el de Alprazolam (25,8 \%) y Bromazepam (22,6 \%) es ligeramente superior en hombres.

Los antidepresivos más utilizados son Escitalopram (16,9\%), Mirtazapina $(14,1 \%)$ y Sertralina $(11,3 \%)$. En este caso, el grupo de Otros Antidepresivos $(47,9 \%)$ e Inhibidores de la Recaptación de Serotonina (45,1 \%) suponen el 93,0 \% de prescripciones. Este último está formado principalmente por Escitalopram (37,5\%) y Sertralina $(25,0 \%)$. Mirtazapina es el más utilizado (29,4\%), dentro del grupo de Otros Antidepresivos. Desvenlafaxina (10,8 \%), Duloxetina (10,8 \%), Escitalopram (10,8 \%), Mirtazapina (10,8 \%) y Sertralina (10,8 \%) son los más prescritos en combinación con ansiolíticos o benzodiacepinas hipnóticas, estando presentes en el 54,0 \% de casos. Por otro lado, Mirtazapina (42,9\%), Trazodona $(35,7 \%)$ y Desvenlafaxina $(28,6 \%)$ son los más prescritos en combinación entre antidepresivos (Figura 3). 
LAÍNA OYAGUE LÓPEZ Y RAMONA MATEOS CAMPOS

PERFIL DEL PACIENTE AMBULATORIO EN TRATAMIENTO

CON ANSIOLÍTICOS Y/O ANTIDEPRESIVOS

Figura 3. Uso de Fármacos Antidepresivos (\%).

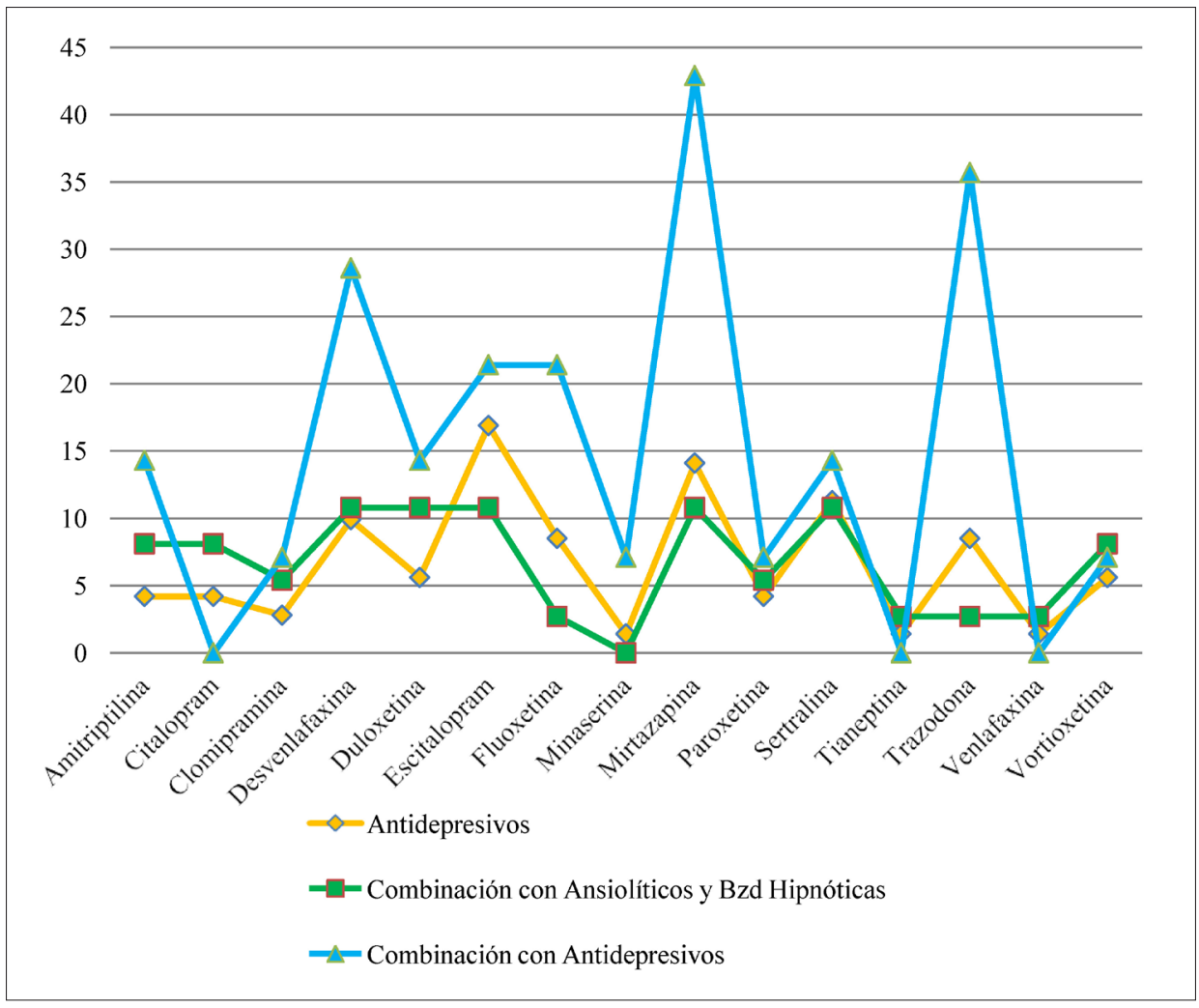

Destaca la mayor utilización de Escitalopram (23,5 \%), Mirtazapina (17,6 \%), Clomipramina $(11,8 \%)$ y Paroxetina $(11,8 \%)$ por los hombres.

\subsection{Ansiolíticos y benzodiacepinas hipnóticas}

Las combinaciones entre fármacos ansiolíticos o benzodiacepinas hipnóticas más utilizadas son las que incluyen benzodiacepinas ansiolíticas e hipnóticas $(53,8 \%)$.

Las indicaciones más frecuentes para la combinación de benzodiacepinas ansiolíticas e hipnóticas son: insomnio $(38,5 \%)$ y ansiedad (30,8 \%). La principal indicación para las combinaciones de dos benzodiacepinas ansiolíticas es el tratamiento de ansiedad (23,1\%), aunque también son utilizadas en el Trastorno Límite de la Personalidad (TLP) (7,7\%), dolor músculo-esquelético $(7,7 \%)$ e 
insomnio $(7,7 \%)$. La indicación recogida para la combinación de benzodiacepinas hipnóticas, ansiolíticas e Hidroxicina es la ansiedad (7,7\%).

Al considerar las indicaciones en función del fármaco, observamos que el tratamiento del insomnio (39,4\%), ansiedad (37,5\%) así como su tratamiento conjunto $(9,6 \%)$ resulta lo más frecuente. Otras indicaciones son el dolor músculoesquelético (2,9\%), TLP (2,9\%), vértigo (1,9\%) y colon irritable (1,0\%), que también señalan otros estudios (García et al., 2010; Kanchan et al., 2012; Aparicio et al., 2006; Caballero et al., 2013). Lorazepam es el más utilizado para tratar la ansiedad (13,5\%) e insomnio $(20,1 \%)$, a pesar de no considerarse hipnóticosedante. Alprazolam está indicado principalmente en ansiedad (13,5\%), siendo con Lorazepam el más empleado. Bromazepam es el segundo más utilizado para el insomnio (4,8 \%), junto con Lormetazepam (4,8 \%) y Zolpidem (4,8 \%). Diazepam es el principal fármaco prescrito para el dolor músculo-esquelético $(1,9 \%)$.

Respecto al modo de uso, la mayoría se prescriben de forma crónica $(85,5 \%$ ), siendo el más utilizado Lorazepam (43,3\%). Los principales fármacos prescritos de forma aguda son Bromazepam (57,1 \%) y Alprazolam (28,7 \%).

Las dosis utilizadas se ajustan a las recomendadas (AEMPS, 2018), siendo la dosis media la más frecuente, excepto para Lormetazepam y Zolpidem, donde lo es la dosis máxima. Las dosis recomendadas de Alprazolam se cumplen en todos los casos, mientras que para Lorazepam ocurre en el 64,3 \%.

\subsection{Antidepresivos}

La combinación entre antidepresivos más frecuente es la de Inhibidores de la Recaptación de Serotonina junto con Otros Antidepresivos (50,1 \%), seguida de combinaciones entre fármacos de este último grupo $(28,6 \%)$.

Las principales indicaciones son la depresión (45,1\%) y ansiedad $(45,1 \%)$, destacando la capacidad de algunos, como Trazodona (8,5 \%), para tratar el insomnio, como señala la literatura (Caballero et al., 2013; Álamo et al., 2016; Contreras, 2013). También, son útiles para tratar el TLP (4,2 \%) y dolor neuropático (4,2 \%), como reflejan algunos estudios (García et al., 2010; Flórez et al., 2010).

Todas las dosis utilizadas se ajustan a lo recomendado (AEMPS, 2018), siendo la dosis recomendada la más frecuente en el caso de Escitalopram (58,3 \%) y Mirtazapina (90,0\%).

\subsection{Efectos adversos y duración del tratamiento}

El 19,1 \% de pacientes en tratamiento con ansiolíticos o benzodiacepinas hipnóticas dice haber sufrido algún efecto adverso, siendo el 13,5\% en el caso de antidepresivos y 24,3\% para pacientes tratados con combinación. 
Alteraciones en el estado anímico (29,4 \%) y somnolencia (29,4 \%) fueron los efectos adversos más frecuentes para ansiolíticos, seguidos de alteraciones gastrointestinales $(23,5 \%)$ y manifestación conjunta de los primeros efectos $(17,6 \%)$.

Los efectos adversos mayoritarios para antidepresivos fueron ansiedad $(28,6 \%)$ y alteraciones gastrointestinales $(28,6 \%)$, seguidos de mareos $(14,3 \%)$ o manifestación conjunta de varios efectos $(28,6 \%)$.

La frecuencia de aparición de efectos adversos en tratamiento con ansiolíticos es mayor entre los 60-69 años (47,1\%), en tratamiento con antidepresivos ocurre entre los 50-59 años (57,1\%) y entre los 30-59 años (66,6 \%) para pacientes tratados con combinación.

La duración del tratamiento es más de dos años en el 64,0 \% de pacientes tratados con ansiolíticos, a pesar de las recomendaciones de no superar las 12 semanas (AEMPS, 2018), en el 51,9\% de los tratados con antidepresivos y el $40,5 \%$ en combinación. Los pacientes de nuevo diagnóstico suponen el 14,6 \% para el grupo de ansiolíticos y el 13,5 \% para el grupo de antidepresivos y combinación. Por otro lado, el 84,6\% de los pacientes asegura cumplir siempre el tratamiento.

\subsection{Pacientes de nuevo diagnóstico}

Los pacientes de nuevo diagnóstico son principalmente mujeres mayores de 50 años para todos los casos. Cabe destacar los estudios superiores como los más frecuentes para el grupo de ansiolíticos $(38,5 \%)$ y la práctica de otra actividad para todos los grupos. Los trabajadores en activo (42,9\%), jubilados $(53,8 \%)$ y amas de casa $(40,0 \%)$ resultan las actividades más frecuentes.

\subsection{Patologías y medicación adicional}

El 76,9 \% de los pacientes padece, al menos, otra patología. La hipercolesterolemia $(32,7 \%)$, hipertensión arterial $(31,7 \%)$ y patologías cardiacas $(16,3 \%)$ son las más frecuentes en todos los tratamientos. Los fármacos hipocolesterolemiantes son los más utilizados (32,7\%), seguidos de antihipertensivos (ARAII 16,3 \%, IECA 7,7 \%; Diuréticos 6,7 \%, Amlodipino 6,7 \%). El dolor es uno de los síntomas más frecuentes (19,2\%), siendo su principal tratamiento AINEs y Tramadol (29,8\%), que, junto con otros fármacos, algunos estudios relacionan con depresión (Mazen et al., 2018). 
LAÍNA OYAGUE LÓPEZ Y RAMONA MATEOS CAMPOS

PERFIL DEL PACIENTE AMBULATORIO EN TRATAMIENTO

CON ANSIOLÍTICOS Y/O ANTIDEPRESIVOS

\section{Conclusiones}

1. El perfil de pacientes en tratamiento con ansiolíticos o benzodiacepinas hipnóticas es mujer de 70 o más años, con estudios secundarios, asalariada y actualmente jubilada.

2. El perfil de pacientes en tratamiento con antidepresivos es mujer de 50-59 años, con estudios primarios, asalariada y trabajadora en activo.

3. El perfil de pacientes tratados con combinación corresponde a mujeres mayores de 50 años, con estudios primarios o secundarios, asalariadas y actualmente jubiladas.

4. Las benzodiacepinas son el grupo farmacológico más prescrito, principalmente Lorazepam, tanto solo como en combinación con antidepresivos u otros ansiolíticos. El hipnótico más utilizado es Lormetazepam.

5. Escitalopram es el antidepresivo más prescrito y con Sertralina, Duloxetina, Desvenlafaxina y Mirtazapina son los más utilizados en combinación con fármacos ansiolíticos. Este último junto con Trazodona son los más utilizados en combinación con otros antidepresivos.

6. En general, las dosis prescritas para todos los medicamentos del estudio se ajustan a lo recomendado en la ficha técnica y los efectos adversos se señalaron en baja proporción.

7. El perfil de pacientes de nuevo diagnóstico es similar al de pacientes en tratamiento de larga duración, siendo la práctica de otra actividad la profesión más frecuente en todos los grupos.

8. Las patologías concomitantes más frecuentes son hipercolesterolemia e hipertensión, junto con su respectivo tratamiento farmacológico.

9. El consumo de ansiolíticos y antidepresivos es más frecuente en mujeres y aumenta con la edad, siendo mayor el consumo de ansiolíticos en personas jubiladas y el de antidepresivo en trabajadoras en activo, y, en general, los tratamientos son de larga duración.

\section{Bibliografía}

AEMPS: Agencia Española de Medicamentos y Productos Sanitarios [Internet]. Madrid: AEMPS; c2013 [citado el 9 de junio 2018] Centro de Información Online de Medicamentos; Fichas Técnicas. Disponible en: https://cima.aemps.es/cima/publico/ home.html

AEMPS: Agencia Española del Medicamento y Productos Sanitarios. Observatorio del Uso de Medicamentos. Utilización de Medicamentos Ansiolíticos e Hipnóticos en España Durante el Periodo 2000-2012. [Internet]. Madrid: AEMPS; 2014 [actualizado 27 enero 2014; citado 10 febrero 2018] Disponible en: https://www.aemps.gob.es/ medicamentosUsoHumano/observatorio/docs/ansioliticos_hipnoticos-2000-2012.pdf

Ediciones Universidad de Salamanca / @@ $\quad$ FarmaJournal, vol. 4, núm. 2 (2019), pp. 19-29 
AEMPS: Agencia Española del Medicamento y Productos Sanitarios. Observatorio del Uso de Medicamentos. Utilización de Medicamentos Antidepresivos en España Durante el Periodo 2000-2013. [Internet]. Madrid: AEMPS; 2015 [actualizado 14 enero 2015; citado 10 febrero 2018] Disponible en: https://www.aemps.gob.es/ medicamentosUsoHumano/observatorio/docs/antidepresivos-2000- 2013.pdf

Álamo González C, Alonso Álvarez ML, Cañellas Dols F, Martín Águeda B, Pérez Díaz H, Romero Santos-Tomás O et al. Pauta de Actuación y Seguimiento: Insomnio. Madrid: Sociedad Española del Sueño; 2016.

Aparicio Basuri, Arbesú Prieto J, Guerrero Iturralde A, Iglesias Rivero R, Martínez Acebal A, Noriega Morán M et al. Guía de Recomendaciones Clínicas: Ansiedad. Asturias: Consejería de Sanidad y Servicios del Principado de Asturias; 2006.

Caballero Navarro A, Fernández Rodríguez I, Fernández Rodríguez M, García García L, García Haro J, García Velasco G et al. Guía de Recomendaciones Clínicas: Depresión. Asturias: Consejería de Sanidad y Servicios Sanitarios del Principado de Asturias; 2013.

Contreras A. Insomnio: Generalidades y Alternativas Terapéuticas de Última Generación. Rev. Med. Clin. Condes. 2013; 24(3):433-441.

Flórez S, León M, Torres M, Reyes F, Serpa JC, Ríos AM. Manejo Farmacológico del Dolor Neuropático. Rev. Col. Anest. 2010; 37(4):356-372.

García López MT, Martín Pérez MF, Otín Llop R. Tratamiento Integral del Trastorno Limite de la Personalidad. Rev. Asoc. Esp. Neuropsiq. 2010; 30(106):263-278.

Kanchan RS, Manmohan S. Current Perspectives in the Pharmacotherapy of Vertigo. Otorhinolaryngol Clin Int J. 2012; 4(2):81-85.

Lorenzo P, Moreno A, Leza JC, Lizasoain I, Moro MA, Portolés A. Velázquez. Farmacología Básica y Clinica. 18th ed. Madrid: Médica Panamericana; 2009.

Mazen Qato D, Ozenberg K, Olfson M. Prevalence of Prescription Medications with Depression as a Potencial Adverse Effect Among Adults in the United States. JAMA. 2018; 319 (22):2289-2298.

Piédrola Gil. Medicina Preventiva y Salud Pública. 12th ed. Barcelona: Elsevier; 2015.

WHO Collaborating Centre for Drug Statistics Methodology. [Internet] Oslo: WHO; c2009 [actualizado 15 enero 2018; citado 6 de febrero 2018] ATC/DDD Index. Disponible en: https://www.whocc.no/atc_ddd_index/

Ediciones Universidad de Salamanca / @@ఠ $\quad$ FarmaJournal, vol. 4, núm. 2 (2019), pp. 19-29 